\title{
Bigger is not always better: anesthetic management of a very large atrial mass
}

\begin{abstract}
Atrial myxomas are the most common primary intracardiac tumors. Only rarely do they arise from the right atrium with a variable clinical presentation. We report on the anesthetic management of an extremely large myxoma arising in the right atrium which had significant extension into both the inferior vena cava (IVC) and superior vena cava (SVC). This case poses unique challenges to both surgeons and anesthesiologists because of the potential for cardiovascular collapse with induction, bleeding, emboli, and significant postoperative complications. With careful preoperative optimization including fluid resuscitation and vigilant monitoring, this patient was able to have a safe anesthetic for her surgical removal of this complex mass.
\end{abstract}

Keywords: echocardiography, heart neoplasms, myxoma, cardiac surgery
Volume 7 Issue 2 - 2016

\author{
Wendy K Bernstein, Christine Ayoola, \\ Kristen Jordan \\ Associate Professor, Division of Cardiothoracic Anesthesiology, \\ University of Maryland School of Medicine, USA
}

Correspondence: Wendy K Bernstein, University of Maryland School of Medicine, 22 S Greene Street S I I C Baltimore Maryland 2|20I, USA, Tel 4I0-328-6I20, Fax 4|0-428-553|, Email wbernstein@anes.umm.edu

Received: October 28,2016 | Published: November 22, 2016
Abbreviations: EKG, electrocardiogram; ABG, arterial blood gas; TEE, transesophageal echocardiography; EF, ejection fraction; INR, international normalized ratio; FFP, fresh frozen plasma; PRBC, packed red blood cells; CT, computerized tomography; POD, postoperative day

\section{Introduction}

Cardiac myxomas are the most common type of primary cardiac tumors with the majority $(>70 \%)$ originating from the left atrium and a smaller percentage from the right atrium $(<18 \%))^{1,2}$ We present an interesting case of a very large atrial tumor that occupied most of the space in the right atrium and was near obstructing both the inferior vena cava (IVC) and superior vena cava (SVC). Anesthetic management was challenging due to the potential for cardiovascular collapse with induction, bleeding, emboli, and significant postoperative complications. With careful preoperative optimization including fluid resuscitation and vigilant monitoring, this patient was able to have a safe anesthetic for her surgical removal of this complex mass.

\section{Case presentation}

A 68yr old female $(175 \mathrm{~cm}, 78.3 \mathrm{~kg})$ presented to an outside hospital with increasing dyspnea on exertion and chest discomfort for approximately a month. On electrocardiogram, she was found to be in atrial fibrillation and was noted to have a large right atrial mass as an incidental finding. She was transferred to University hospital for further workup. Her medical history was significant for hypotension, depression, asthma and hypothyroidism. Her medications included wellbutrin, levothyroxine, singulair, symbicort and prednisone. Her laboratory exam demonstrated a hemoglobin of 11.2grams per deciliter.

Preoperative workup included a cardiac catheterization which showed normal coronaries and a carotid duplex which was normal. Transthoracic echocardiogram demonstrated a large mass which filled the entire right atrium and obstructed the inferior vena caval flow. A magnetic resonance imaging (MRI) showed a right atrial mass measuring $7.6 \times 6.4 \times 6.4$ centimeters which was highly suggestive of a right atrial hemangioma. Computerized tomogram angiography (CTA) abdomen showed a right atrial mass with internal serpiginous vessels but without involvement of the renal vein and initially interpreted as an angiosarcoma. Urine and plasma metanephrines were negative. The patient was anticoagulated with heparin and scheduled for removal of her heart mass.

The patient was hemodynamically stable. Vital signs on presentation to the operating room were $178 / 77 \mathrm{~mm} \mathrm{Hg}$, pulse $84 \mathrm{bpm}$ and oxygen saturation $95 \%$ on room air. On examination, she was awake and alert, oriented to person, place and time. The patient had no murmurs noted on physical examination. Electrocardiogram (EKG) showed normal sinus rhythm.

There was great concern regarding safe induction of anesthesia due to a near complete obstruction of superior vena cava (SVC). The patient was volume loaded with 500milliliters of 5\% albumin, 1.5liters of crystalloid and 2 units of packed red blood cells. A right radial arterial line was placed preinduction. General anesthesia was delivered carefully using a induction regimen consisting of etomidate/ fentanyl/ midazolam and rocuronium for muscle relaxation. Surgeons were present in the operating room for induction. After induction of general anesthesia, a multiplane transesophageal echocardiography probe was inserted into the esophagus without difficulty. Transesophageal echocardiography revealed a very large mass in the severly dilated right atrium; the attachment point was not completely clear, but may have involved the atrial septum. The interatrial septum was intact with no evidence for an atrial septal defect. There was also mild to moderate tricuspid regurgitation. Left ventricle was normal in size with ejection fraction $55 \%$ (Figures $1 \& 2$ ). The patient remained hemodynamically stable throughout the case.

The patient underwent resection of a right atrial mass, and tricuspid valve repair with a \# 28 Edwards Lifesciences annuloplasty ring via a median sternotomy. Cannulation was performed via the ascending aorta and bicaval cannulation. The IVC was cannulated using a seldinger technique from the right common femoral vein using a 25 Fr venous cannula at $30 \mathrm{~cm}$ which was placed just into the inferior vena cava by TEE visualization. The SVC was cannulated with a $24 \mathrm{Fr}$ venous cannula. When the activated clotting time (ACT) was greater than 480seconds, full cardiopulmonary bypass was initiated. The aorta was crossclamped and a liter of cold antegrade cardioplegia was administered with prompt arrest of the heart. The right atrial mass was easily dissected from the atrial wall in entirety, with no residual lesions noted. The tricuspid valve was noted to be grossly dilated and 
8 centimeters in length. Pathology of the right atrial mass demonstrated a myxoma (Figure 3 ). Total bypass time was 50 minutes and aortic cross clamp was 38 minutes. The patient received a total of 3 units of fresh frozen plasma, and 750milliliters of cell saver. Post bypass TEE demonstrated trace tricuspid regurgitation, complete removal of atrial mass with no residual, and no atrial septal defect. Her ejection fraction was $55 \%$. Postoperative course was complicated by atrial fibrillation for which the patient was started on beta blocker and aspirin. She was discharged home on postoperative day five.

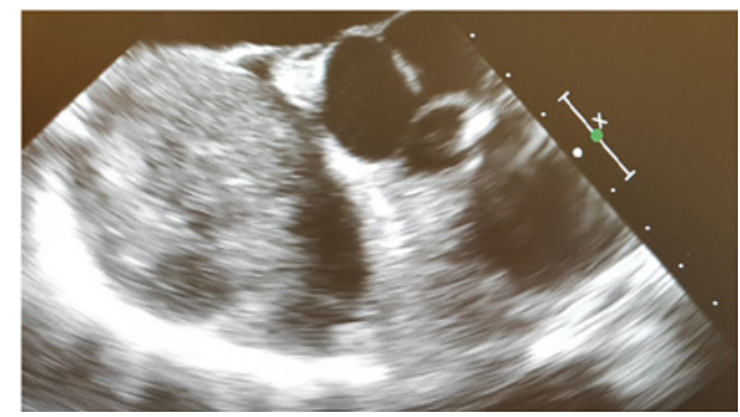

Figure I Transesophageal echocardiography image of a midesophagea modified 5 chamber view shows a very large heterogeneous mass which occupies the majority of the right atrium and with extension into the inferior vena cava.

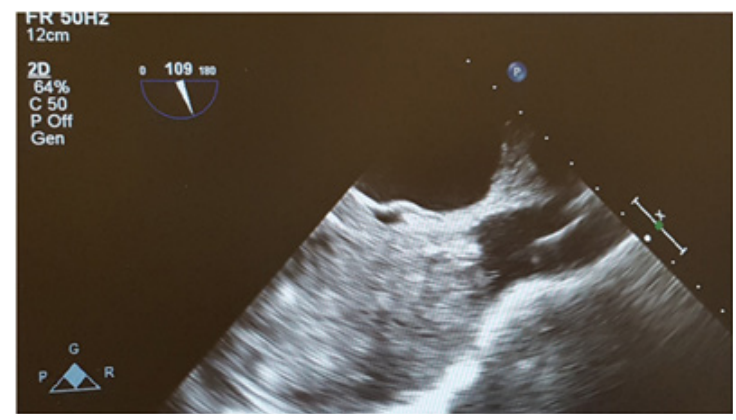

Figure 2 Transesophageal echocardiography midesophageal modified bicaval view demonstrates an extremely large right atrial mass with extension into the superior vena cava.

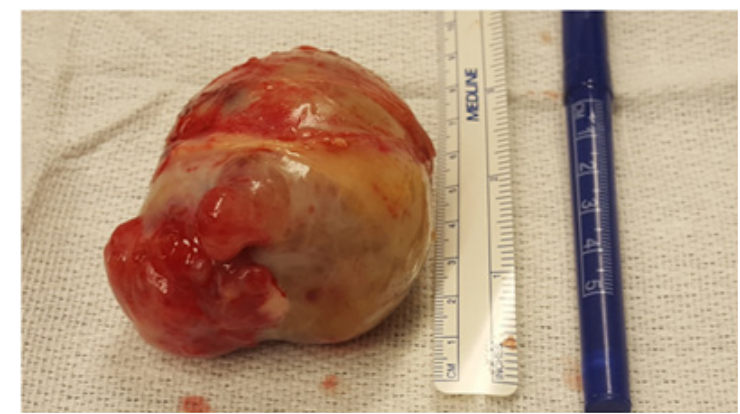

Figure 3 Photograph shows the very large excised right atrial mass which was attached via a wide base (arrow).

\section{Discussion}

Atrial myxomas are the most common primary intracardiac tumors and only rarely do they arise from the right atrium with a variable clinical presentation. ${ }^{1,2}$ It is not uncommon for them to be asymptomatic until they achieve a very large size, as demonstrated in this case. ${ }^{3}$ Great care needed to be taken during induction to prevent cardiovascular collapse. In this case, this was accomplished with volume loading this patient with crystalloid and colloid, as well as two units of PRBC (packed red blood cells). Without doing this, we believe that the negative inotropic effects of the anesthetic agents combined with the drop in systemic vascular resistance that is frequently seen with induction would have had catastrophic consequences. The surgeons were immediately available in the operating room during induction in the event that the patient needed to be emergently placed on cardiopulmonary bypass. The use of TEE was important to locate the location of the mass and determine the extent of involvement (Kumar , Zini, Komanapalli). ${ }^{4-6}$ TEE was also beneficial in visualizing the mass during cannulation, to facilitate introduction of the cannulas and prevent embolization by fragmentation.

\section{Conclusion}

There are very few reports of an atrial mass of this size with extension into the inferior vena cava and superior vena cava. Careful anesthetic planning and preparation, understanding the potential for cardiovascular collapse with induction, anticipation for major blood loss and potential for emboli, are all necessary for a successful outcome. TEE proved useful in the perioperative evaluation of the location of the mass and determination of its extension into both the inferior vena cava and superior vena cava. This is valuable information which can determine location of cannulation sites.

\section{Acknowledgments}

None.

\section{Conflicts of interest}

Author declares there is no conflicts opf interest.

\section{Funding}

None.

\section{References}

1. Darwazah AK, Eida M, Baltrawy M. Myxoma at junction of inferior vena cava and right atrium. Tex Heart Inst J . 2011;38(5):591-593.

2. Choi $\mathrm{CH}$, Park $\mathrm{CH}$, Kim JS, et al. Giant biatrial myxoma nearly obstructing the orifice of the inferior vena cava. J Cardiothor Surg. 2013;8:148.

3. Buyahater M, Aktunc E. Giant left atrial myxoma causing mitral valve obstruction and pulmonary hypertension. Can J Surg. 2008;51(4):E97-98.

4. Kumar K, Basker S, Jeslin L, et al. Anesthetic management for removal of adrenocortical carcinoma with thrombus in the inferior vena cava extending to the right atrium. J Anaesth Clin Pharma. 2011;27(4):571-573.

5. Zini L, Haulon S, Leroy X, et al. Endoluminal occlusion of the inferior vena cava in renal cell carcinoma with retro- or suprahepatic caval thrombus. BJU Int . 2006;97(6):1216-1220.

6. Komanapalli CB, Tripathy U, Sokoloff M, et al. Intraoperative renal cell carcinoma tumor embolization to the right atrium: Incidental by transesophageal echocardiography. Anesth Analg. 2006;102(2):378-379. 